\title{
EFEITO DA PODA, RALEIO DE FRUTOS E USO DE FITORREGULADORES NA PRODUÇÃO DE TANGERINEIRAS (Citrus deliciosa Tenore) cv. MONTENEGRINA ${ }^{1}$
}

\author{
IVAR ANTONIO SARTORI ${ }^{2}$, OTTO CARLOS KOLLER ${ }^{3}$, SERGIOMAR THEISEN ${ }^{2}$, PAULO VITOR DUTRA DE SOUZA $^{4}$, \\ RENAR JOÃO BENDER ${ }^{4}$, GILMAR ARDUINO BETTIO MARODIN ${ }^{4}$
}

RESUMO - O experimento aqui relatado teve o propósito de estudar a poda de ramos, raleio de frutos e uso de fitorreguladores para diminuir a alternância de produção e melhorar a qualidade físico-química dos frutos de tangerineiras 'Montenegrina' (Citrus deliciosa Tenore). São plantas enxertadas sobre laranjeira 'Caipira'(Citrus sinensis L. Osbeck) e estão em um pomar comercial de 6 anos de idade, da empresa Panoramas Citrus, situada no município de Butiá-RS, na latitude $29^{\circ} 57^{\prime} \mathrm{S}$ e longitude $51^{\circ} 40^{\prime} \mathrm{W}$. Neste experimento, foram realizados os seguintes tratamentos: A) Testemunha ${ }^{\circ} 1$ : plantas com carga excessiva; B) Testemunha $\mathrm{n}^{\circ} 2$ : plantas sem carga; C) Raleio manual de $66 \%$ dos frutos em plantas com carga excessiva, em fevereiro; D) Pulverizações de plantas excessivamente carregadas, utilizando-se de 200 mg.L $\mathrm{L}^{-1}$ de Ethrel (24\% ethephon), em novembro; E) Poda de frutificação, em plantas excessivamente carregadas, em dezembro; F) Idem "E" acrescido de raleio manual de $33 \%$ dos frutos, em fevereiro; G) Idem "E" acrescido de pulverização com 50 mg.L.-1 de 2,4-DP (95\% de 2,4 diclorofenoxipropiônico), no final da queda natural de frutos, em dezembro; H) Poda de plantas com alternância de produção, em dezembro; I) Idem "H" acrescido de pulverização com 15 mg.L L $^{-1}$ de ácido giberélico (10\% de $\mathrm{AG}_{3}$ ), em maio. $\mathrm{O}$ delineamento experimental foi o de blocos casualizados, com parcelas subdivididas no tempo, tendo 4 repetições e 3 plantas úteis por parcela. A poda diminuiu a produção por planta no ano de excessiva carga de frutos e reduziu a alternância de produção, quando associada à prática do raleio manual de $33 \%$ dos frutos. Em plantas alternantes a poda foi mais eficiente para quebrar a alternância de produção. A poda e o raleio manual de $66 \%$ aumentaram a massa média e melhoraram a qualidade dos frutos, mas o raleio manual de $66 \%$ dos frutos, somente, foi insuficiente para quebrar a alternância de produção. A $200 \mathrm{mg} \mathrm{L}^{-1}$, o ethephon não exerceu ação de raleio de frutos. O 2,4-DP com pulverização de $50 \mathrm{mg} \mathrm{L}^{-1}$ não modificou o tamanho dos frutos. $\mathrm{O} \mathrm{AG}_{3}$, na concentração de $10 \mathrm{mg} \mathrm{L}^{-1}$, aplicado em maio, não inibiu a diferenciação floral.

Termos para indexação: Citrus deliciosa, poda, raleio manual, reguladores de crescimento.

\section{PRUNING EFFECT, HAND THINNING AND USE OF GROWTH REGULATORS ON 'MONTENEGRINA' MANDARIN ORANGES (Citrus deliciosa Tenore)}

\begin{abstract}
The experiment had the purpose to evaluate the effects of pruning, hand thinning and growth regulators sprays to decrease the production alternation and increase the physic-chemical quality of 'Montenegrina' mandarin oranges (Citrus deliciosa Tenore). They are plants budded on 'Caipira' Orange (Citrus sinensis L. Osbeck), and the present study was conducted in a six-yearold commercial orchard, in Panoramas Citrus Company, located in Butiá-RS, Southern of Brazil (29 $57^{\prime}$ 'S - 51 $40^{\circ}$ 'W). The following treatments were applied: A) Control 1, trees with heavy fruit load; B) Control 2, trees without bearing fruits; C) Hand thinning of 66\% of the fruits on heavy loaded trees, in February; D) $200 \mathrm{mg} \mathrm{L}^{-1}$ Ethrel sprays of heavy loaded trees; E) Pruning of heavy loaded trees in December; F) Pruning of heavy loaded trees plus hand thinning in 33\% of the fruit; G) Pruning of heavy loaded trees plus 50 mg L${ }^{1}$ of 2,4-DP sprays (95\% of 2,4 dichlorofenoxipropionic), at the end of the natural fruit fall in December; $\mathrm{H}$ ) Pruning of alternate bearing trees in December; I) Pruning of alternate bearing trees plus $10 \mathrm{mg} \mathrm{L}^{-1} \mathrm{GA}_{3}$ giberelic acid sprays (10\% of $\left.\mathrm{AG}_{3}\right)$ in May. The treatments were applied in a randomized blocks design with 4 replicates and 3 plants as experimental unit. Pruning decreased the production in each plant in a year of excessive fruit quality, and diminished the alternate bearing of trees associated to the hand thinning practice of $33 \%$ of the fruits. In alternate plants, the hand thinning was more efficient to break the production alternation. Hand thinning and pruning of $66 \%$ increases the mean mass and better the fruit quality, but the hand thinning of $66 \%$ of the fruits was only enough to break the production alternation. Ethephon sprays showed no thinning effect with concentrations of $200 \mathrm{mg} \mathrm{L}^{-1}$ and $10 \mathrm{mg} \mathrm{L}^{-1}$ of GA 3 sprays didn't inhibit bud flower differentiation and $50 \mathrm{mg} \mathrm{L}^{-1} 2,4-\mathrm{DP}$ sprays have no effect on increasing fruit size.
\end{abstract}

Index terms: Citrus deliciosa, pruning, hand thinning, growth regulators.

\footnotetext{
(Trabalho 09-2006). Recebido em 20-01-2006. Aceito em 03-12-2006. Parte de trabalho de tese de doutorado financiado pela FAPERGS, CAPES e CNPq ${ }^{2}$ Eng $^{\circ}$. Agr ${ }^{\circ}$. Mestre em Fitotecnia, aluno de doutorado do Programa de Pós-Graduação em Fitotecnia da UFRGS. Av. Bento Gonçalves 7712 , Bairro Agronomia, Cx.P 776, CEP 91501970. Porto Alegre-RS. E mail: ivar@ufrgs.br

${ }_{3}^{3}$ Dr. Prof. Convidado da Faculdade de Agronomia da UFRGS, Bolsista 1A do CNPq. Endereço: Rua Largo Setembrina, 126 Viamão-RS, CEP 94415-400, ockoller@adufrgs.ufrgs.br.

${ }^{4}$ Dr. Prof. Adjunto da Faculdade de Agronomia da UFRGS, Bolsista de Produtividade de Pesquisa do CNPq.
} 


\section{INTRODUÇÃO}

A produção mundial de citros foi de, aproximadamente, 108 milhões de toneladas em 2004, sendo o Brasil, nos últimos anos, o maior produtor, com mais de 19 milhões de toneladas (FAO, 2005). O Rio Grande do Sul é o quinto maior produtor do País, sendo que, segundo dados do IBGE, em 2005, a produção gaúcha alcançou 545.172 toneladas, em uma área de 42.556 hectares, que representou receita, para os citricultores, de 145 milhões de reais (João, 2004).

O cultivo da tangerineira 'Montenegrina' tem apresentado grande expansão no Rio Grande do Sul devido à produção tardia e aos frutos de boa qualidade. Por outro lado, essa variedade apresenta o inconveniente da alternância de produção, caracterizada por um ano de grande carga de frutos pequenos, seguido de outro com pequena ou insignificante produção (Marodin, 1986; Nienow et al., 1991; Souza et al., 1991; Schwarz et al., 1992; Rodrigues et al., 1998).

A poda de frutificação é uma prática pouco recomendada por reduzir a produtividade e o crescimento da planta (Koller, 1994); contudo, quando se desejam frutas de boa qualidade para consumo in natura, a poda é uma prática importante (Panzenhagen et al., 1992; Miozzo et al., 1992 e Rodriguez \& Villalba, 1998) e contribui para a aeração da planta, facilidade de raleio manual de frutos, colheita e execução de tratamentos fitossanitários.

Para a produção de frutos de mesa em pequenas propriedades do Rio Grande do Sul, com o uso da mão-de-obra familiar, é mais recomendável a prática do raleio manual (Schwarz et al., 1992; Nienow et al., 1991 e Rodrigues et al., 1998), que consiste na retirada do excesso de frutos ainda jovens, os quais podem ser vendidos a indústrias, para extração de óleos essenciais da casca.

Por sua vez, o raleio químico com pulverizações com Ethrel (24\% de ethephon), em concentrações entre 200 a 300 mg.L ${ }^{-1}$, realizado durante a plena queda natural, tem promovido a formação de elevada percentagem de frutos grandes e diminuído a alternância de produção na cultivar Montenegrina (Marodin, 1986; Souza et al., 1991). Em contrapartida, apresenta algumas desvantagens, como: permanência de frutos no interior da copa, devido às baixas pressões de pulverização; diminuição do teor de sólidos solúveis totais no suco dos frutos e queda de folhas. A inibição da diferenciação de gemas florais, com aplicação de ácido giberélico $\left(\mathrm{AG}_{3}\right)$ no período de repouso vegetativo, é outro método bastante utilizado na Espanha (Agustí \& Almela, 1991; Sanches, 2000) para evitar o excesso de produção.

Também, na Espanha (Agustí, 1996; Guardiola \& Garcia, 2000), aplicações de fitorreguladores como 2,4-DP (ácido 2,4diclorofenoxipropiônico), aplicados após a plena queda natural dos frutinhos, estimularam o aumento do tamanho dos frutos em tangerineiras 'Clementinas' e 'Satsumas'.

O propósito deste trabalho foi de estudar o efeito da poda sobre a produção, em comparação com técnicas de raleio de frutos já pesquisadas anteriormente, na Faculdade de Agronomia da Universidade Federal do Rio Grande do Sul, para quebrar ou diminuir a alternância de produção e melhorar a qualidade dos frutos produzidos por tangerineiras 'Montenegrina'.

\section{MATERIAL E MÉTODOS}

$\mathrm{O}$ experimento foi realizado num pomar da empresa Panoramas Citrus, situada no município de Butiá-RS, junto à BR290. Foram utilizadas plantas da cv. Montenegrina (Citrus deliciosa Tenore), com altura média de 2,30 m e 6 anos de idade, enxertadas sobre laranjeira 'Caipira' (Citrus sinensis L. Osbeck), plantadas no espaçamento de 3 x 6 metros, em um solo Argissolo Vermelho distrófico típico, EMBRAPA, 1999). O clima da região é classificado como Cfa, ou seja, temperado sem estação seca definida.

O delineamento experimental foi o de blocos ao acaso, com parcelas subdivididas no tempo, com nove tratamentos, quatro repetições e usando três plantas úteis por parcela. Os tratamentos constituíram-se de: A) Testemunha "1" (plantas excessivamente carregadas), sem poda, sem raleio de frutos e sem uso de fitorreguladores; B) Testemunha "2" (plantas sem carga), sem poda, sem raleio de frutos e sem uso de fitorreguladores; C) Raleio manual de $66 \%$ dos frutos de plantas com carga excessiva, em janeiro e fevereiro, sem poda; D) Pulverizações de plantas excessivamente carregadas com 200 mg. $\mathrm{L}^{-1}$ de ethephon, em novembro/dezembro, durante a plena queda natural de frutos; E) Poda de frutificação, de plantas excessivamente carregadas, em dezembro, suprimindo galhos para promover a entrada de luz e retirando de 30 a $40 \%$ dos ramos produtivos terminais nos galhos remanescentes, sem raleio de frutos; F) Poda de plantas excessivamente carregadas, conforme tratamento "D", e raleio manual de $33 \%$ dos frutos, preferencialmente na periferia da copa; G) Poda de plantas excessivamente carregadas, conforme tratamento "D", e pulverização com $50 \mathrm{mg} . \mathrm{L}^{-1}$ de 2,4-DP, no final da queda natural de frutos, sem fazer raleio de frutos; $\mathrm{H}$ ) Poda de plantas sem frutos (em alternância de produção), conforme tratamento "D"; I) Poda conforme tratamento "D" de plantas sem frutos, e pulverização com 10 a 15 mg. $\mathrm{L}^{-1}$ de $\mathrm{AG}_{3}$, entre 10 e 20 de maio (para raleio de flores).

Os tratos culturais que não foram objeto de avaliação, tais como: adubações, controle de pragas e moléstias, manejo e cobertura do solo, foram uniformes em todos os tratamentos.

$\mathrm{O}$ raleio manual de frutos, em plantas não-podadas, para eliminação de $66 \%$ dos frutos, foi feito retirando-se duas frutas de cada três existentes e procurando-se deixar uma fruta a cada $15 \mathrm{~cm}$ de comprimento do ramo frutífero. Para a eliminação de $33 \%$, foi retirada uma fruta de cada três existentes.

Os fitorreguladores foram aplicados com pulverizador costal, com pressão que variou entre 70 e 80 lb/pol, devido à dificuldade de mantê-la constante com esse equipamento. A solução foi aplicada até que ocorresse o início do escorrimento, das folhas para o solo, gastando-se, em média, três litros por planta.

O ethephon foi utilizado sob a forma de produto comercial Ethrel (24\% de ethephon), pulverizado na fase de plena queda natural de frutinhos; o ácido giberélico $\left(\mathrm{AG}_{3}\right.$ a $\left.10 \%\right)$, na forma de PRO-GIB ${ }^{\circledR}$,foi aplicado para indução de gemas floríferas, na primeira quinzena de maio, e o 2,4-DP (95\% de 2,4 diclorofenoxipropiônico, DICLORPROP ${ }^{\text {) }}$ foi aplicado após a queda natural dos frutos, no mês de novembro, para aumento de 
tamanho de frutos.

Antes da colheita, foi realizada a coleta de raízes para determinar o teor de substâncias de reservas totais. Por ocasião da colheita, os frutos de cada árvore foram contados, pesados e classificados em três classes de calibres comerciais. No Laboratório de Fisiologia Pós-Colheita da Faculdade de Agronomia da UFRGS, foram realizadas análises para a determinação dos teores de substâncias de reserva contidas nas raízes das plantas, pelo método de Priestley, descrito por Souza (1990).

Os dados experimentais obtidos foram submetidos a análises de variância segundo modelo de delineamento de blocos casualizados, com parcelas subdivididas, e utilizando o teste F, ao nível de 5\% de probabilidade, para testar a significância das diferenças.

\section{RESULTADOS E DISCUSSÃO}

Com relação à quebra de alternância de produção, os dados apresentados na Tabela 1 evidenciam que as plantas dos tratamentos-Testemunha "1" (com carga excessiva em 2001) e as que haviam sido pulverizadas com ethephon (tratamento D), não apresentavam flores em 2002, nem frutos nos ramos marcados. Isto foi consequiência da elevada carga de frutos que as plantas desses tratamentos haviam produzido em 2002, como pode ser observado na Tabela 2.

Também, por sua vez, se refletiu no baixo teor de substância de reserva das raízes (Tabela 3). Esses resultados mostram que, na concentração de $200 \mathrm{mg} . \mathrm{L}^{-1}$, o ethephon não exerceu raleio de frutos, diferentemente do raleio de $57 \%$ que havia sido observado por Marodin (1986) na mesma cultivar, com porta-enxerto Poncirus trifoliata Raf., em solo arenoso e mantido limpo.

A ausência de efeito do ethephon, neste experimento, talvez possa ter sido conseqüência de diferenças de solo, fatores meteorológicos, porta-enxerto, manejo do solo, vigor das plantas e outros fatores que possam ter inibido ou neutralizado a ação de raleio de frutos, comprovada por outros pesquisadores (Marodin, 1986 e Sousa et al., 1991).

Por sua vez, a presença de flores em 31-09-2002 e de frutos, em ramos marcados, antes da queda natural, em 07-11-03 (Tabela 1), bem como o maior número de frutos produzidos em 2003, em relação à testemunha "1", mesmo que sem diferença significativa (Tabela 2), indicam que os tratamentos "C" (raleio manual de 66\%), "F" (poda com raleio manual de 33\% dos frutos) e "H" (poda de plantas alternantes) tiveram tendência de quebrar a alternância de produção, o que foi corroborado pelo aumento no teor de substâncias de reservas das raízes observado principalmente no ano de 2002 (Tabela 3). Deve-se salientar que o raleio de $66 \%$, repetido a cada dois anos, é o indicado para que ocorra a quebra da alternância de produção, segundo dados obtidos por Schwarz et al. (1992) e Rodrigues et al. (1998).

Ainda, na Tabela 2, pode ser observado que, nos tratamentos "C" (raleio manual de 66\%) e "F" (poda com raleio manual de $33 \%$ dos frutos), o raleio não atingiu os índices desejados de $66 \%$ e $33 \%$, sendo que, efetivamente em 2002, o raleio foi só de $52 \%$ e $24 \%$, e em 2004 , só de $41 \%$ e $21 \%$, respectivamente nos tratamentos $\mathrm{C}$ e F. Isso ocorreu devido à dificuldade de visualizar todos os frutos, pequenos e verdes entre as folhas, nas plantas com copa extremamente fechada, relutância dos operários em se abaixarem para retirar os frutos da parte inferior da planta e receio de retirar frutos em excesso. Essa constatação também foi observada por Nienow et al. (1991). Verifica-se, pois, que existe a tendência de fazer-se um raleio de frutos bem menor do que o desejado. Isso pode ter contribuído para que a tendência de quebra da alternância de produção não tivesse atingido valores estatisticamente significativos.

O raleio de $33 \%$ dos frutos em plantas podadas (tratamento F) diminui a produção de frutos, e foi o tratamento mais eficiente na diminuição da alternância de produção (Tabela 2).

O tratamento "H" (poda com alternância em 2001) também exerceu efeito de quebra da alternância, que ficou evidenciado pela menor produção do que a testemunha "2" (planta sem carga em 2001), em 2003, e maior produção em 2004 (Tabela 2). Isso indica que a poda de plantas alternantes pode exercer melhor efeito sobre a quebra da alternância de produção, do que a poda de árvores muito carregadas.

Os demais tratamentos: "E" (poda em plantas muito carregadas) e "G" (poda + 50 mg.L.-1 de 2,4-DP), diminuíram o número de frutos produzidos em 2002, mas o índice de florescimento, em 2002, e a produção de frutos, em 2003, foram baixos, indicando que, para quebrar efetivamente a alternância de produção, a poda em plantas muito carregadas deve ser complementada com raleio manual de frutos (Tabelas 1 e 2).

Comparando resultados com poda em plantas alternantes (tratamento I) + a aplicação de $10 \mathrm{mg} . \mathrm{L}^{-1}$ ácido giberélico, em 12 de maio de 2003 (Tabela 2), verifica-se que o número de frutos vingados em 31-01-03 não diferiu da testemunha "B" (planta sem carga em 2001), indicando que, nesta concentração, o ácido giberélico não inibiu a floração, diferentemente das observações de Agustí \& Almela (1991). Evidências indicam que épocas de aplicação e concentrações de $\mathrm{AG}_{3}$ são variáveis para cada cultivar e local de estudo (Sanches, 2000).

Os tratamentos "E" (poda em plantas muito carregadas), "F" (poda em plantas muito carregadas + raleio manual de 33\% dos frutos) e "G" (poda em plantas muito carregadas + 50 mg.L' ${ }^{1}$ de 2,4 -DP) diminuíram em aproximadamente $40 \%$ a produção por planta em 2002, e o teor de substâncias reservas nas raízes foi superior à testemunha “1”, evidenciando que, pela diminuição da produção, a poda diminuiu o consumo de substâncias reservas nas raízes, reduzindo o esgotamento das plantas e possibilitando, assim, que elas produzissem frutos no ano subseqüente (Tabelas 2 e 3). Esses dados, com três anos consecutivos de podas, estão em conformidade com resultados encontrados por Panzenhagen et al. (1992) e Miozzo et al. (1992), em que a poda de raminhos, feita num só ano, não quebrou totalmente a alternância de produção, necessitando ser feita ao longo dos anos subseqüentes.

Poda e raleio manual aumentaram as reservas nas raízes, sendo que o tratamento que manteve relativamente altos os teores de reservas, durante os três anos, foi a poda associada ao raleio manual de frutos de $33 \%$ (tratamento F) (Tabelas 2 e 3). Pode-se observar que, em 2002, o tratamento-testemunha "1" proporcionou massa média de $80 \mathrm{~kg}$ de frutos produzidos por 
planta, enquanto as podas nos tratamentos de plantas com carga excessivas reduziram a massa dos frutos em praticamente $50 \%$, o que também foi constatado para o número de frutos (Tabela 2). A melhor regularidade da massa de frutos produzidos nos três anos ocorreu no tratamento " $F$ " (poda associada ao raleio manual de $30 \%$ ).

O tratamento "G” (poda +50 mg.L $\left.{ }^{-1} 2,4-D P\right)$ não incrementou a massa de frutos produzidos, pois não diferiu do tratamento "E" (poda em plantas com carga excessiva) em 2002 (Tabela 4), diferentemente das respostas encontradas por Agustí \& Almela (1991), em tangerineira Clementina 'Fina'; Agustí et al. (1996), em tangerineira 'Satsuma Owari'; Polero \& Covatta (1996), em tangerineiras Satsuma cvs. Okitsu e Owari; Duarte et al. (1996), em tangerineiras cv. Esbal. Entretanto, segundo Taiz \& Zeiger (2004), existem evidências de que, em frutos que contêm sementes, as auxinas sintéticas não exercem resposta porque as auxinas presentes no endosperma podem contribuir com o crescimento do fruto durante o estádio inicial, e o embrião em desenvolvimento pode ser a fonte principal de auxina durante os estágios seguintes, como fontes naturais de auxinas para o crescimento do fruto, sendo desnecessário o suprimento da auxina sintética, 2,4-DP, via exógena.

A influência dos tratamentos sobre a produção de frutos classificados em três categorias é melhor salientada nos resultados apresentados na Tabela 5 , onde se verifica que, entre os anos de 2002 e 2004, os tratamentos "E" (poda de plantas com carga excessiva em 2001) e "F" (poda de plantas com carga excessiva + raleio manual de $30 \%$ ) produziram maior quantidade de frutos de primeira categoria do que a testemunha " 1 " (plantas com carga excessiva em 2001) e se equivaleram ao raleio manual de frutos. Pode-se verificar que também foram estes tratamentos (poda de plantas com carga excessiva em 2001) "E" e (poda de plantas com carga excessiva + raleio manual de $30 \%$ ) "F" que proporcionaram a maior quantidade acumulada de frutos de primeira categoria, produzidos nos três anos.

O melhor desempenho das plantas desses tratamentos na produção de frutos de primeira categoria deveu-se à diminuição da carga, que evitou o esgotamento de substâncias reservas, conforme já foi comentado anteriormente.

TABELA 1 - Número de flores e frutos vingados de tangerineiras (Citrus deliciosa Tenore) cv. Montenegrina, submetidas à poda, raleio manual e fitorreguladores. Panoramas Citrus, Butiá-RS. 2002-2003.

\begin{tabular}{|c|c|c|c|c|}
\hline Tratamentos & $\begin{array}{l}\text { Número de } \\
\text { Flores } \\
\text { 31-09-02 }\end{array}$ & $\begin{array}{l}\text { Número de } \\
\text { Frutinhos } \\
\text { retidos } \\
31-01-03\end{array}$ & $\begin{array}{c}\text { Frutos antes } \\
\text { da queda } \\
\text { natural } \\
7-11-03\end{array}$ & $\begin{array}{l}\text { Frutos após a } \\
\text { queda natural } \\
18-12-03\end{array}$ \\
\hline A) Testemunha 1 com carga excessiva em 2001 & e & $0 \quad \mathrm{~b}$ & $28 \mathrm{~cd}$ & $12 \mathrm{a}$ \\
\hline B) Testemunha 2 sem carga em 2001 & $6,70 \mathrm{ab}$ & $1,19 \mathrm{ab}$ & 0 & $0 \mathrm{~b}$ \\
\hline C) Raleio manual de $66 \%$ (fev 02$)$ & $0,70 \quad \mathrm{e}$ & $0,80 \quad b$ & $61 \mathrm{~b}$ & $10 \mathrm{a}$ \\
\hline D) $200 \mathrm{mg} \cdot \mathrm{L}^{-1}$ ethephon (nov/01) & 0 & $0 \quad b$ & $47 \mathrm{bc}$ & $10 \mathrm{a}$ \\
\hline E) Poda (dez/01) c/ carga excessiva em 2001 & 0,90 de & $0,43 \mathrm{ab}$ & $66 \mathrm{ab}$ & $12 \mathrm{a}$ \\
\hline F) Poda (dez) + Raleio manual de 33\% (fev/02) & $3,10 \mathrm{~cd}$ & $1,24 \mathrm{ab}$ & $67 \mathrm{ab}$ & $12 \mathrm{a}$ \\
\hline G) Poda (dez) + 2,4-DP $50 \mathrm{mg} \cdot \mathrm{L}^{-1}(\mathrm{dez} / 01)$ & $0,82 \quad \mathrm{e}$ & $0,48 \mathrm{ab}$ & $88 \mathrm{a}$ & $11 \mathrm{a}$ \\
\hline H) Poda (dez/01) planta s/ carga em 2001 & $8,90 \mathrm{a}$ & $1,80 \mathrm{a}$ & 17 & $8 \mathrm{a}$ \\
\hline I) Poda (idem H) $+\mathrm{AG}_{3} 10 \mathrm{mg} \cdot \mathrm{L}^{-1}$ (maio/02) & $3,71 \mathrm{bc}$ & $1,12 \mathrm{ab}$ & 14 & $7 \mathrm{a}$ \\
\hline $\mathrm{CV} \%$ & 16,2 & 16,7 & 21,1 & 20,2 \\
\hline
\end{tabular}

Médias seguidas por letras distintas, na coluna, diferem entre si, ao nível de 5\% de probabilidade, pelo teste de Tukey.

TABELA 2 - Número de frutos produzidos por planta de tangerineiras (Citrus deliciosa Tenore) cv. Montenegrina, submetida à poda, raleio manual e fitorreguladores. Panoramas Citrus, Butiá-RS. 2002-2004.

\begin{tabular}{|c|c|c|c|c|c|c|c|c|}
\hline \multirow[b]{2}{*}{ Tratamentos } & \multicolumn{6}{|c|}{ Número de frutos produzidos } & \multirow{2}{*}{\multicolumn{2}{|c|}{ Total }} \\
\hline & & 06/ago/2002 & 10/ago/2003 & & 23/ag & 50/2004 & & \\
\hline A) Testemunha 1 com carga excessiva em 2001 & A & $957 \mathrm{~b}$ & B 56 de & & $1101 \mathrm{a}$ & & $2114 \mathrm{a}$ & \\
\hline B) Testemunha 2 sem carga em 2001 & $\mathrm{~B}$ & 18 & A 325 a & $\mathrm{B}$ & 6 & $\mathrm{f}$ & 350 & d \\
\hline C) Raleio manual (RM)de $66 \%$ (fev/02 e 04$)$ & A & $588 \mathrm{c}(647)^{* *}$ & B 114 & A & 743 & $\mathrm{~b}(514) * *$ & $1446 \mathrm{~b}$ & b \\
\hline *D) $200 \mathrm{mg} . \mathrm{L}^{-1}$ ethephon (nov/01) & & $1145 \mathrm{a}$ & B 5 & & & - & & \\
\hline E) Poda (dez/01, 02 e 03) c/ carga excessiva & A & $557 \mathrm{c}$ & В 71 & A & 594 & $\mathrm{c}$ & 1222 & bc \\
\hline F) Poda (idem E) + R.M. de $33 \%($ fev/02 e 04$)$ & A & $428 \mathrm{~d}(135)^{* *}$ & B 142 & A & 490 & $d(128) * *$ & 1060 & $\mathrm{c}$ \\
\hline$\left.{ }^{*} \mathrm{G}\right)$ Poda (idem E) + 2,4-DP $50 \mathrm{mg} \cdot \mathrm{L}^{-1}(\mathrm{dez} / 01)$ & A & $623 \mathrm{c}$ & B 58 & & & - & & \\
\hline H) Poda (dez/01, 02 e 03) s/ carga em 2001 & $\mathrm{~B}$ & 14 & A 210 bc & A & 232 & e & 457 & $\mathrm{~d}$ \\
\hline * I) Poda (idem H) $+\mathrm{AG}_{3} 10 \mathrm{mg} \cdot \mathrm{L}^{-1}$ (maio) & $\mathrm{B}$ & 30 & A $281 \mathrm{ab}$ & & & - & & \\
\hline CV \% tratamento & & & 8,5 & & & & 9,2 & \\
\hline $\mathrm{CV} \%$ ano & & & 11,9 & & & & & \\
\hline
\end{tabular}


TABELA 3 - Porcentagem de substâncias de reserva existentes nas raízes de tangerineiras (Citrus deliciosa Tenore) cv. Montenegrina, submetidas à poda, raleio manual e fitorreguladores. Panoramas Citrus, Butiá-RS. 2002-2004.

\begin{tabular}{|c|c|c|c|}
\hline \multirow[b]{2}{*}{ Tratamentos } & \multicolumn{3}{|c|}{ Teor de substâncias reservas nas raízes (\%) } \\
\hline & Jul/2002 & Jul/2003 & Jul/2004 \\
\hline A) Testemunha 1 com carga excessiva em 2001 & A 16,50 b & A 17,49 & A 18,74 \\
\hline B) Testemunha 2 sem carga em 2001 & A 22,43 a & B 14,59 & A 22,64 \\
\hline C) Raleio manual (RM) de $66 \%$ (fev/02 e 04$)$ & B $17,04 \quad b$ & B 16,20 & A 22,40 \\
\hline D) $200 \mathrm{mg} \cdot \mathrm{L}^{-1}$ ethephon (nov/01) & B 14,67 & A 20,86 & - \\
\hline E) Poda (dez/01, 02 e 03) c/ carga excessiva & A $19,48 \mathrm{ab}$ & A 16,68 & A 17,78 \\
\hline F) Poda (idem E) + R.M. de 33\% (fev/02 e 04) & A 22,35 a & B 17,31 & $\mathrm{AB} 19,13$ \\
\hline G) Poda (idem E) + 2,4-DP 50 mg.L L $^{-1}(\mathrm{dez} / 01)$ & A $21,59 \mathrm{ab}$ & A 20,74 & - \\
\hline H) Poda (dez/01, 02 e 03) s/ carga em 2001 & A 22,25 a & B 16,55 & $\mathrm{AB} 19,52$ \\
\hline I) Poda (idem $\mathrm{H}$ ) $+\mathrm{AG}_{3} 10 \mathrm{mg} . \mathrm{L}^{-1}$ (maio) & A $23,51 \mathrm{a}$ & B 17,99 & - \\
\hline $\mathrm{CV} \%$ tratamento & \multicolumn{3}{|c|}{6,2} \\
\hline $\mathrm{CV} \%$ ano & \multicolumn{3}{|c|}{5,3} \\
\hline
\end{tabular}

Médias seguidas por letras minúsculas iguais na coluna e por letras maiúsculas iguais na linha não diferem entre si, ao nível de 5\% de probabilidade, pelo teste de Tukey. - Dados não coletados em 2004.

TABELA 4 - Massa total dos frutos produzidos por planta (kg) de tangerineira (Citrus deliciosa Tenore) cv. Montenegrina, submetidas à poda, raleio manual e fitorreguladores. Panoramas Citrus, Butiá-RS. 2002-2004.

\begin{tabular}{|c|c|c|c|c|}
\hline \multirow[b]{2}{*}{ Tratamentos } & \multicolumn{3}{|c|}{ Massa total dos frutos (kg) } & \multirow[b]{2}{*}{ total } \\
\hline & 2002 & 2003 & 2004 & \\
\hline A) Testemunha 1 com carga excessiva em 2001 & A $81,01 \mathrm{a}$ & $\begin{array}{lll}\mathrm{B} & 5,71 \mathrm{de}\end{array}$ & A 85,43 a & $172,15 \mathrm{a}$ \\
\hline B) Testemunha 2 sem carga em 2001 & B $2,14 \quad d$ & A 29,31 a & B 0,57 & 32,02 \\
\hline C) Raleio manual (RM) de $66 \%$ (fev/02 e 04$)$ & A $60,00 \quad b$ & $\mathrm{~B} 12,00 \mathrm{~cd}$ & A $65,31 \mathrm{~b}$ & $137,31 \mathrm{~b}$ \\
\hline *D) $200 \mathrm{mg} \cdot \mathrm{L}^{-1}$ ethephon (nov/01) & A 87,84 a & B 0,51 & - & \\
\hline E) Poda (dez/01, 02 e 03$) \mathrm{c} /$ carga excessiva & A $55,94 \mathrm{~b}$ & B 7,14 & A 58,67 bc & $121,71 \mathrm{c}$ \\
\hline F) Poda (idem E) + R.M. de $33 \%($ fev/02 e 04$)$ & A $45,70 \quad c$ & B 14,43 bc & A 52,08 c & 109,71 \\
\hline$\left.{ }^{*} \mathrm{G}\right)$ Poda (idem E) + 2,4-DP 50 mg.L $\mathrm{L}^{-1}(\mathrm{dez} / 01)$ & A $60,46 \mathrm{~b}$ & B 6,28 de & - & \\
\hline H) Poda (dez/01, 02 e 03) s/ carga em 2001 & B 1,61 & A $22,11 \mathrm{ab}$ & A 25,77 & 49,49 \\
\hline * I) Poda (idem $\mathrm{H})+\mathrm{AG}_{3} 10 \mathrm{mg} \cdot \mathrm{L}^{-1}$ (maio) & B 3,36 & A 29,80 a & - & \\
\hline CV \% tratamento & & 6,4 & & 6,1 \\
\hline $\mathrm{CV} \%$ ano & & 11,5 & & \\
\hline
\end{tabular}

TABELA 5 - Número acumulado de frutos das categorias comerciais: primeira, segunda, primeira + segunda e terceira categoria de tangerineiras (Citrus deliciosa Tenore) cv. Montenegrina, submetidas à poda, raleio manual e fitorreguladores. Panoramas Citrus, Butiá-RS. 2002-2004.

\begin{tabular}{|c|c|c|c|c|}
\hline \multirow[b]{2}{*}{ Tratamentos } & \multicolumn{4}{|c|}{$\begin{array}{l}\text { Número acumulado de frutos de cada categoria, produzidos } \\
\text { nos anos de } 2002 \text { a } 2004\end{array}$} \\
\hline & $1^{\mathrm{a}} \mathrm{Cat}$ & $2^{\text {a }}$ Cat & $1^{\mathrm{a}}+2^{\mathrm{a}} \mathrm{Cat}$ & $3^{\text {a }}$ Cat \\
\hline A) Testemunha 1 com carga excessiva em 2001 & $21 \mathrm{~d}$ & 845 a & $866 \mathrm{~b}$ & $1199 \mathrm{a}$ \\
\hline B) Testemunha 2 sem carga em 2001 & $16 \mathrm{~d}$ & 167 & 183 & 151 \\
\hline C) Raleio manual (RM) de $66 \%$ (fev/02 e 04$)$ & $73 \mathrm{bc}$ & 851 a & $924 \mathrm{a}$ & $485 \mathrm{~b}$ \\
\hline *D) $200 \mathrm{mg} \cdot \mathrm{L}^{-1}$ ethephon (nov/01) & - & - & - & - \\
\hline E) Poda (dez/01, 02 e 03) c/ carga excessiva & $95 \mathrm{ab}$ & 825 a & $920 \mathrm{a}$ & $266 \mathrm{c}$ \\
\hline F) Poda (idem E) + R.M.de $33 \%($ fev/02 e 04$)$ & $101 \mathrm{a}$ & 754 a & $855 \mathrm{~b}$ & 180 \\
\hline$\left.*^{*} \mathrm{G}\right)$ Poda (idem E) + 2,4-DP 50 mg.L $\mathrm{L}^{-1}(\mathrm{dez} / 01)$ & - & - & - & - \\
\hline H) Poda (dez/01, 02 e 03) s/ carga em 2001 & $59 \mathrm{c}$ & $298 \mathrm{~b}$ & $357 \mathrm{c}$ & 86 \\
\hline * I) Poda (idem H) $+\mathrm{AG}_{3} 10$ mg. $\mathrm{L}^{-1}$ (mai) & - & - & - & - \\
\hline $\mathrm{CV} \%$ & 16,4 & 9,1 & 7,7 & 18,6 \\
\hline
\end{tabular}

Médias seguidas por letras distintas iguais, na coluna, não diferem entre si, ao nível de 5\% de probabilidade, pelo teste de Tukey. * Dados não coletados em 2004 


\section{RESULTADOS}

Os resultados obtidos neste experimento são apresentados nas Tabelas de número 1 até 5, que se seguem.

\section{CONCLUSÕES}

Nas condições em que foi realizada esta pesquisa e com três anos de avaliação, pode-se concluir que:

1- A poda anual diminui a produção, mas melhora a qualidade dos frutos e diminui a alternância de produção.

2- O raleio manual de $66 \%$ dos frutos não diminui a alternância de produção, mas melhora a qualidade dos frutos.

3- Na concentração de $200 \mathrm{mg} . \mathrm{L}^{-1}$, o ethephon não exerce efeito de raleio de frutos.

4- A poda anual, acrescida de $50 \mathrm{mg} . \mathrm{L}^{-1}$ 2,4-DP, não incrementa o tamanho do fruto, mas intensifica a cor da casca.

5- Na concentração de $10 \mathrm{mg} \cdot \mathrm{L}^{-1}, \mathrm{o} \mathrm{AG}_{3}$ não inibe a diferenciação floral.

\section{REFERÊNCIAS}

AGUSTÍ, M.; ALMELA, V. Aplicación de fitorreguladores en citricultura. Barcelona: AEDOS, 1991. $261 \mathrm{p}$.

AGUSTÍ, M.; ORENGA, V.A.; MÁS, J.P. Tratamientos para aumentar el tamaño del fruto em los agrios: Valencia: generalitat valenciana. Conselleria d'Agricultura i Pesca, 1996.79p.

DUARTE, A.M.M.; TRINDADE, D.T.G.; GUARDIOLA, J.L. Thinning of 'Esbal' clementine with 2,4dichlorophenoxyacetic acid. Influence on yield, fruit size and fruit quality. Proceedings of the Interamerican Society for Tropical Horticultural, Alexandria, v. 2, s/n, p. 929-933, 1996.

EMBRAPA. Centro Nacional de Pesquisa de Solos. Sistema brasileiro de classificação de solos. Brasília, Rio de Janeiro, 1999. 412p.

FAO. Produção mundial de frutas cítricas. 2005. Disponível em: <http://www.fao.org>. Acesso em: 11 jan. 2005.

GUARDIOLA, J.L.; GARCÍA, L. Increasing fruit size in Citrus. Thining and stimulation of fruit growth. Plant Growth regulation, Dordrecht, v. 31, p. 121-132, 2000.

IBGE. Produção Vegetal. Agricultura: laranja, limão e tangerina, 2005. Disponível em: <http://www.ibge.org.br>. Acesso em: 11 jan. 2005.

JOÃO, P.L. (Coord.). Levantamento da fruticultura comercial do Rio Grande do Sul. Porto Alegre: EMATER/RS-ASCAR, 2004. $89 \mathrm{p}$.

KOLLER, O. C. Citricultura: laranja, limão e tangerina. Porto Alegre: Rigel, 1994. 446p.

MARODIN, G.A.B. Raleio químico e manual de frutinhos em tangerineira (Citrus deliciosa Tenore) cv. Montenegrina. 1986. 124f. Dissertação (Mestrado, Programa de Pósgraduação em Fitotecnia) - Faculdade de Agronomia, Universidade Federal do Rio Grande do Sul, Porto Alegre, 1986.
MIOZZO, A.K.; KOLLER. O.C.; SCHWARZ, S.F.; PANZENHAGEMN, N.V. Efeito da poda de ramos e do raleio manual de frutos sobre a produção de tangerineiras 'Montenegrina'. Revista Brasileira de Fruticultura, Cruz das Almas, v.14, n.2, p. 59-63, 1992.

NIENOW, A.A.; KOLLER. O.C.; SCHWARZ, S.F.; MARODIN, G.A.B.; RIBOLDI, J. Efeito da intensidade e épocas de raleio manual de frutos sobre a produção de tangerineiras 'Montenegrina'. Revista Brasileira de Fruticultura, Cruz das Almas, v.13, n.1, p. 35-40, out. 1991.

PANZENHAGEN, N.V.; KOLLER. O.C.; SCHWARZ, S.F.; MIOZZO, A.K. Efeito da poda e raleio de frutos jovens sobre a produção de tangerineiras 'Montenegrina'. Revista Brasileira de Fruticultura, Cruz das Almas, v.14, n.2, p. 53$58,1992$.

POLERO, J.H.; COVATTA, F. Efecto del 2,4-DP sobre el tamaño y massa de frutos de mandarinos satsuma (citrus unshiu, Marcovitch), cvs. Okitsu y Owari. Revista Brasileira de Fruticultura, Cruz das Almas, v. 18, n. 1, p. 137-146, 1996.

RODRIGUES. L.R.; SCHWARZ, S.F.; RECKZIEGEL, V.P.; KOLLER, O.C. Raleio manual de frutos em tangerinas 'Montenegrina'. Pesquisa Agropecuária Brasileira, Brasília, v.33, n. 8, p. 14-21. 1998.

RODRÍGUEZ, J.J.P.; VILLALBA, D.B. Poda de los cítricos. Valência: Generalitat valenciana: conselleria de agricultura, pesca y alimentacion, 1998. 15p. (Série citricultura, 2).

SANCHES, F.R. Aplicação de biorreguladores vegetais: aspectos fisiológicos e aplicações práticas na citricultura mundial. Jaboticabal: Funep, 2000. 160p.

SCHWARZ, S.F.; KOLLER. O.C.; NIENOW, A.A. Intensidades e épocas de raleio manual em tangerineiras 'Montenegrina'. Pesquisa Agropecuária Brasileira, Brasília, v. 27, n. 8, p. 41-48, 1992.

SOUZA, P.V.D de. Efeito de concentrações de etefon e pressões de pulverização foliar no raleio de frutinhos em tangeineiras (Citrus deliciosa Tenore) cv. Montenegrina. 1990. 139f. Dissertação (Mestrado) - Programa de Pós-Graduação em Fitotecnia, Faculdade de Agronomia, Universidade Federal do Rio Grande do Sul, Porto Alegre, 1990.

SOUZA, P.V.D.de.; KOLLER. O.C.; SCHWARZ, S.F. Efeito do raleamento químico e de pressões de pulverização na qualidade dos frutos de tangerineiras (Citrus deliciosa Tenore) cv. Montenegrina. Revista Brasileira de Fruticultura, Cruz das Almas, v. 13, n. 1, p. 99-105, 1991.

TAIZ, L.; ZEIGER, E. Fisiologia vegetal. Porto Alegre: Artmed, 2004. 719p.

Rev. Bras. Frutic., Jaboticabal - SP, v. 29, n. 1, p. 005-010, Abril 2007 